\title{
Correction to: Superior biomechanical properties and tying time with the modified Prusik knot and Wittstein suture loop to the Krackow stitch
}

\author{
Chih-Kai Hong ${ }^{1} \cdot$ Cheng-Li Lin ${ }^{1,2}$. Jui-Ming Yang ${ }^{1,3} \cdot$ Chien-An Shih ${ }^{1} \cdot$ Yi-Chuan Chou ${ }^{1}$ Ming-Long Yeh ${ }^{2,4}$. \\ Chen-Hao Chiang ${ }^{4,5} \cdot$ Wei-Ren Su ${ }^{1,2,6}$
}

Received: 30 October 2017 / Published online: 20 November 2017

(c) Springer-Verlag GmbH Germany, part of Springer Nature 2017

\section{Correction to: Arch Orthop Trauma Surg (2017) https://doi.org/10.1007/s00402-017-2830-8}

Due to an error during the production process, the legend of Fig. 1 was incorrect. The correct legend of Fig. 1 should be
"Photographs and schematics of the a modified Prusik knot and b Wittstein suture loop."
The original article can be found online at https://doi. org/10.1007/s00402-017-2830-8.

\footnotetext{
Wei-Ren Su

suwr@ms28.hinet.net

Chih-Kai Hong

yayahong@gmail.com

Cheng-Li Lin

jengli94@yahoo.com.tw

Jui-Ming Yang

jmyang25@gmail.com

Chien-An Shih

sam5214330@hotmail.com

Yi-Chuan Chou

groupuie@yahoo.com.tw

Ming-Long Yeh

mlyeh@mail.ncku.edu.tw

Chen-Hao Chiang

chiangabaca@gmail.com
} 